\title{
Incidence of Pectoralis Major Injuries Has Increased 40\% Over the Last 22 National Football League Seasons
}

\author{
Blake M. Bodendorfer, M.D., Steven F. DeFroda, M.D., Henry T. Shu, B.S., \\ Derrick M. Knapik, M.D., Daniel S. Yang, B.S., and Nikhil N. Verma, M.D.
}

\begin{abstract}
Purpose: To examine trends in the incidence of pectoralis major (PM) injuries over the last 22 National Football League (NFL) seasons and identify risk factors for injuries requiring operative management. Methods: Publicly available data from the 1998-1999 through 2019-2020 NFL seasons were reviewed to identify PM injuries, demographics, injury mechanisms, and management. Injury incidence was calculated using linear regression per 10,000 athlete-exposures, while risk factors for operative management were identified through multivariate logistic regression. Results: There were 258 PM injuries. Mean athlete age at the time of injury was 27.1 years (range: 21-37) with a mean body mass index of 32.6 (range: 24.8-43.1). Overall incidence was 0.603 per 10,000 athlete-exposures, which was found to significantly increase with time by 0.039 per athlete-exposures per year $\left(R^{2}=.787, P<.001\right)$. Defensive athletes accounted for $64.7 \%$ of PM injuries. Repair was performed in 48.8\% of athletes, with defensive linemen (odds ratio [OR] 3.78, CI 1.42-10.60, $P=.009$ ), defensive backs (OR 12.20, CI 2.13-76.60, $P=.006$ ), linebackers (OR 8.98, CI 2.58-33.60, $P<.001$ ), more recent time of injury (OR 1.11, CI 1.05-1.17, $P<.001$ ), and shorter NFL experience (OR .77 for older athletes, CI .59-.99), $P=.047)$ at significant risk for operative treatment. Conclusion: A total of 258 PM injuries were identified over 22 NFL seasons, with an overall incidence of 0.603 per 10,000 athlete-exposures, which was found to increase by 0.039 injuries per 10,000 athlete-exposures per year. Repair was performed in $48.8 \%$ of athletes, with more recent time of injury, shorter NFL experience, defensive linemen, defensive backs and linebackers at significantly higher risk for operative treatment. Study Design: Cohort study; Level of evidence, 3
\end{abstract}

$\mathbf{T}$ he incidence of pectoralis major (PM) tears has increased over the last three decades as the number of athletes participating in all levels of American football continues to rise. ${ }^{1-5}$ PM tears has been reported to occur primarily at the bone-tendon interface when the arm is extended and externally rotated while under maximal contraction. ${ }^{6-10}$ In athletes, the majority of documented PM tears have occurred during weight

From Midwest Orthopaedics at Rush, Chicago, Illinois, U.S.A. (B.M.B., S.F.dF., D.M.K., N.N.V.); School of Medicine, The Johns Hopkins University, Baltimore, Maryland, U.S.A. (H.T.S.); and Warren Alpert Medical School, Brown University, Providence, Rhode Island, U.S.A. (D.S.Y.).

The authors report the following potential conflicts of interest or sources of funding: N.N.V. is a board member at the American Orthopaedic Society for Sports Medicine, the American Shoulder and Elbow Surgeons, and the Arthroscopy Association of North America. N.N.V. is a member of the editorial board of Knee and SLACK, Inc. N.N.V. has received research support from Arthrex, Inc., Breg, Ossur, Smith $\theta$ Nephew, Wright Medical Technology, Inc., and Vindico Medical-Orthopedics Hyperguide. N.N.V. has received stock options from Cymedica, Omeros, and Minivasive. N.N.V. is a paid consultant for Minivasive and Orthospace. N.N.V. has received royalties from Arthroscopy, Smith $\theta$ Nephew, and Vindico Medical-Orthopedics Hyperguide. Full lifting, primarily bench press; however, direct trauma to the arm and chest has also been reported as a less frequent cause of tears. ${ }^{8,9,11}$ At the professional level of competition, the ever-increasing size and speed of athletes further elevate the risk of PM tear. ${ }^{12}$

Although not essential for everyday function, the integrity of the pectoralis major in elite football athletes is important to ensure full athletic potential. ${ }^{7,13}$ As a

ICMJE author disclosure forms are available for this article online, as supplementary material. None of the other authors have any other relevant financial disclosures.

Received December 19, 2020; accepted March 29, 2021.

Address correspondence to Blake M. Bodendorfer, MD, 1611 W. Harrison St., Midwest Orthopaedics at Rush, Chicago, IL 60612 USA. E-mail: BlakeBodendorfer@gmail.com

(C) 2021 THE AUTHORS. Published by Elsevier Inc. on behalf of the Arthroscopy Association of North America. This is an open access article under the CC BY-NC-ND license (http://creativecommons.org/licenses/by-nc-nd/4.0/). 2666-061X/202026

https://doi.org/10.1016/j.asmr.2021.03.019 
dynamic stabilizer of the shoulder, ${ }^{7}$ the pectoralis major serves as a powerful adductor, internal rotator, and flexor of the humerus. ${ }^{14}$ As such, an intact muscle and tendon are required to provide maximal strength and range of motion to meet the position-specific needs in American football athletes. ${ }^{13}$ Despite the increase in PM injury incidence, no investigation has quantified the yearly increase in PM injuries.

The purpose of this investigation was to examine trends in the incidence of pectoralis major (PM) injuries over the last 22 NFL seasons and identify risk factors for injuries requiring operative management. We hypothesized there would be an overall low incidence that increased with time, with the majority of injuries occurring in defensive athletes, with these same athletes having the highest risk for operative management.

\section{Methods}

\section{Data Collection}

Publicly available data were reviewed to identify all reported PM injuries occurring in NFL athletes between the 1998-1999 and 2019-2020 seasons, ${ }^{15,16}$ using previously reported data collection methods. ${ }^{17-22}$ NFL seasons were grouped into two evenly distributed time periods: Period l (1998-1999 through 2008-2009) and Period 2 (2009-2010 through 2019-2020), as the NFL implemented substantial changes in tackling rules during Period 2. ${ }^{23}$ These rule changes made tackles involving helmet-initiated contact illegal, as well as low tackles where the defender initiated a roll or lunge. ${ }^{23}$ Moreover, defenders were allowed to swipe, wrap, or grab a passer below the knees without receiving a foul. ${ }^{23}$ Inclusion criteria were NFL athletes identified as having sustained a PM injury, using publicly available data, while exclusion criteria were athletes that had sustained upper extremity injuries not explicitly classified as PM injuries.

Initial data collection was independently performed by two authors [HTS and BMB] by querying publicly available data from online league and player databases for weekly regular season NFL injury reports and identifying injuries reported as "pectoral", "chest", and "shoulder". ${ }^{15,16}$ NFL injury reports consist of practice reports documenting athlete availability during NFL practices, along with game status reports that capture athlete availability during games. ${ }^{24}$ All NFL teams are mandated by the league to publish injury reports after each regular season practice and prior to each regular season game. $^{24}$ Pro-Football-Reference.com (Sports Reference LLC, Philadelphia, PA) was also used to identify all "pectoral", "chest", and "shoulder" injuries that resulted in a player being designated as injured reserve (IR) or physically unable to perform (PUP). ${ }^{24}$ All athletes initially identified as having sustained a PM injury were further verified by reviewing publicly available press releases. Following the two independent authors' data collection, identified athletes were compared, and any disagreements in athlete inclusion were resolved through mutual discussion with the first author.

For athletes meeting inclusion criteria, athlete characteristics at the time of injury (age, body mass index [BMI], years of experience, and position) were recorded. Injury characteristics, including injury setting (Combine, preseason/offseason, regular season, postseason), activity (practice/workout versus in-game injury), injury classification (grades I-III; strain, partial tear, and complete tear), as well as management (nonoperative versus operative) were recoded when available.

\section{Data Analysis and Statistical Methods}

Incidence of PM injuries per 10,000 athlete-exposures was calculated on the basis of previously reported methodology by assuming 53 players per team, 105 practices per season, 16 games per season, and 32 teams in the NFL. ${ }^{25}$ Changes in league size were also accounted for, with only 30 teams in the 1998-1999 season and 31 teams in the 1999-2000 and 2000-2001 seasons. An athlete-exposure was defined as previously described as a single participation in a single practice or game. $^{25,26}$ Differences in injury incidence between Period 1 and Period 2 was also calculated.

Normal distribution of the data was checked via the Shapiro-Wilk normality test. A Student's $t$-test was used to determine statistical significance between incidence rates between Period 1 and Period 2. To identify the incidence rate trend over the course of the study, a simple linear regression was performed with Pearson's product moment correlation coefficient $(r)$ calculated to identify the strength of linear association. Comparisons between time points for each cohort were evaluated using a 2-tailed paired $t$-tests to compare players against themselves. Risk factors for operative injury were identified through multivariate logistic regression controlling for athlete age, BMI, years of NFL experience, position, year of injury, and injury setting. The offensive line was used as the reference, as that position had the greatest number of offensive injuries. Relationship between grade of tear and operative versus nonoperative treatment was assessed through $\chi^{2}$-ana/lu tlysis. Statistical analysis was performed using RStudio V1.1.442 (RStudio, Inc., Boston, MA). Player characteristics were expressed as mean (range), while odds ratios (OR) were expressed with $95 \%$ confidence intervals (CI). An $\alpha$ value of .05 was used.

\section{Results}

A total of 258 PM injuries were identified in 245 NFL athletes between 1998 and 2020. Mean athlete age at the time of injury was 27.1 (range: 21.0-37.0) years, 
while mean BMI was 32.6 (range: 24.8-43.1). Eleven athletes were reported to sustain 2 separate PM injuries, while 1 athlete experienced 3 PM injuries. Four of those reinjuries were contralateral PM injuries. In the 1 player that had 3 injuries, the second injury was ipsilateral, and the last injury was contralateral. The other 7 repeat injuries did not have public reports of whether those injuries were contralateral or ipsilateral. Complete tears were the most commonly reported injury type (Table 1).

Defensive athletes sustained the greatest number of injuries $(64.7 \%)$, with linebackers being the most commonly injured athlete based on position, followed by offensive linemen (Table 2).

As a percentage of total injuries across the years of the study, injuries were most frequently reported as occurring during games $(76.0 \%, n=196$ of 258$)$, followed by practice/workouts $(22.5 \%, n=58$ of 258$)$, while $1.6 \%(n=4$ of 258$)$ of injuries occurred at the NFL Combine. Of the total injuries across the years of the study, $69.3 \%(n=176$ of 258$)$ occurred during NFL regular season games or practice, $29.1 \%(n=58$ of 258$)$ occurred during the preseason games or practice, and $1.2 \%(n=3$ of 258$)$ occurred during postseason games or practice. The overall incidence of injuries during Combines was 122 per 10,000 athlete-exposures, assuming, on average, 327 athletes attended the combine per year. The incidence of injuries occurring during practices was 0.142 per 10,000 athleteexposures, and the incidence of injuries occurring during regular season games was 3.158 per 10,000 athlete-exposure. The overall injury incidence during the 22 NFL seasons analyzed was 0.603 per 10,000 athlete-exposures, while the incidence was found to increase with increasing year $\left(R^{2}=.787\right)$ (Fig 1$)$.

When comparing injury incidence between Period 1 (1998-2008) versus Period 2 (2009-2019), the incidence of PM injuries was noted to significantly increase from 0.395 per 10,000 athlete-exposures during Period 1 to 0.893 per 10,000 athlete-exposures in Period 2 $(P<.001)$. A linear increase in the annual incidence over the entire study period was calculated at 0.039 per 10,000 athlete-exposures per year. When checking for normal distribution via the Shapiro-Wilk test, incidences from Period $1(P=.361)$ and incidences from Period $2(P=.843)$ were not significantly different than the normal distribution.

A total of $45 \%$ of athletes with PM injuries were nonoperatively managed compared to $48.8 \%$ treated operatively (Table 3 ). Treatment could not be determined in $16(6.2 \%)$ of injuries. There was no significant difference in age $(P=.194)$ or BMI $(P=.779)$ between operative and nonoperative injuries. Risk factors predictive of athletes undergoing operative management for PM injuries were more recent year of injury, fewer years of NFL experience at the time of injury, defensive
Table 1. Distribution of Pectoralis Major Injuries based on Tear Degree

\begin{tabular}{lcc}
\hline \multicolumn{1}{c}{ Tear Degree } & Number of Injuries $(\%)$ & Incidence \\
\hline Strain (Grade I) & $90(34.9)$ & 0.210 \\
Partial tear (Grade II) & $33(12.8)$ & 0.077 \\
Complete tear (Grade III) & $132(51.2)$ & 0.309 \\
Unknown & $3(1.2)$ & N/A \\
\hline
\end{tabular}

Table 1 shows the number and incidence of pectoralis major injuries by tear degree. Incidence is reported per 10,000 athleteexposures. N/A, not applicable.

linemen, defensive backs, and linebackers (Table 4). Of grade 3 tears, $100 \%(n=120)$ were operative, whereas $18.5 \%(n=5)$ of grade 2 tears were operative and $100 \%(n=89)$ of grade 1 tears were nonoperative $(P<.001)$.

\section{Discussion}

Over 22 NFL seasons, there was 258 PM injuries in 245 NFL athletes, with a strong and significant linear increase in injury incidence with more recent NFL seasons. Repair was performed in $49 \%$ of athletes, with more recent year at the time of injury, shorter NFL experience, defensive linemen, defensive backs, and linebackers being predictive of athletes undergoing operative management. Complete tears were the most commonly reported, and PM injuries occurred at higher frequency in defensive athletes, with linebackers possessing the highest incidence, followed by offensive linemen. Although the NFL Injury Surveillance System (ISS) is considered the gold standard for NFL injury epidemiology research, we consider the use of public data to be generally accurate. The use of multiple public databases with weekly injury reports and press releases used in our study allowed us to capture the greatest number of PM injuries, a methodology that has been used in multiple investigations. ${ }^{17-22}$

Following the implementation of an NFL rule change penalizing helmet-initiated contact, a significantly increased incidence of PM injuries was reported. The increased incidence could be related to the change in tackling technique forcing athletes to rely more on open arm tackles, placing the arm in a vulnerable position for PM injuries. However, linear regression analyses between those two periods showed an increase of linear annual incidence change per year of $0.036\left(R^{2}=\right.$ $.386)$ to $0.047\left(R^{2}=.655\right)$ per athlete-exposure per year. Thus, it is likely that the increase between the two time periods may be due to the overall linear increase in annual incidence of 0.039 per athlete-exposure per year $\left(R^{2}=.787\right)$ that we identified instead of relating to the tackling rule changes between these 2 periods. As such, further investigations evaluating for potential factors accounting for the increased incidence of PM injuries is warranted. 
Table 2. Injury Distribution by Player Positions

\begin{tabular}{lc}
\hline \multicolumn{1}{c}{ Position } & Number of Pectoralis Major Injuries (\%) \\
\hline Defense & $\mathbf{1 6 7}(\mathbf{6 4 . 7 )}$ \\
Defensive lineman & $50(29.9)$ \\
Linebacker & $73(43.7)$ \\
Defensive back/secondary & $44(26.3)$ \\
Offense & $\mathbf{9 0 ( 3 4 . 9 )}$ \\
Offensive lineman & $54(60.0)$ \\
Running back & $12(13.3)$ \\
Wide receiver & $7(7.8)$ \\
Tight end & $9(10.0)$ \\
Quarterback & $8(8.9)$ \\
Special teams & $\mathbf{1}(\mathbf{0 . 4 )}$ \\
\hline
\end{tabular}

Table 2 shows the number of pectoralis major injuries that occurred within each player position.

Overall, the incidence of PM injuries was noted to increase with increasing year. This finding has been previously reported in other investigations. Tarity et al. identified 10 complete PM ruptures across 10 seasons of the NFL using the NFL ISS, ${ }^{27}$ with $50 \%$ of injuries occurring in defensive players, generally while tackling. Moreover, $90 \%$ of PM ruptures occurred during games. ${ }^{27}$ An incidence of 0.004 for complete tears per season across the study period was reported, lower than our incidence of 0.174 injuries per 10,000 athleteexposures between 2000 and 2010. Recently, Sahota et al. similarly used the NFL ISS to examine the incidence of all PM injuries, including strains, partial tears, and complete ruptures, from the 2000-2014 NFL seasons. ${ }^{25}$ The authors identified an overall incidence of PM injuries across the 15 -season period to be .688 per 10,000 athlete-exposures, slightly greater than the annual incidence of 0.603 per 10,000 athlete-exposures across 22 seasons in our investigation. When using our data from the 2000-2014 seasons, the incidence across the 15 seasons was slightly lower at .502 per 10,000 athleteexposures. However, Sahota et al. $^{25}$ and Tarity et al. ${ }^{27}$ used the NFL ISS, a league-wide electronic medical records system that documents all injuries occurring in athletes for medical and safety research. As such, we expect a degree of variability to exist between these studies and ours regarding the exact number of injuries per season, as we anticipate PM injuries to be recorded in the NFL ISS, which were not publicly disclosed.

Pectoralis major injuries were most frequently reported to occur during in-game competition, with the overall incidence during games to be 3.158 per 10,000 athlete-exposures versus 0.142 per 10,000 athleteexposures during practice. However, the highest incidence of PM injury per athlete-exposure was during the NFL Combine, at 122 PM injuries per 10,000 athleteexposures. This may be related to the requirement of NFL Combine participants to bench press 225 pounds for a maximum number of repetitions. Previous reports of pectoralis major tears in elite-level athletes cite injuries occurring during weightlifting, especially the bench press. ${ }^{8,9,28}$ In their series examining 17 athletes with pectoralis major rupture, Schepsis et al. ${ }^{9}$ found bench press accounted for $47 \%$ of injuries, while de Castro Pochini et al. ${ }^{28}$ reported injury secondary to bench press in $60 \%(n=12$ of 20$)$ of athletes in their cohort, by far the most common mechanism in both studies. For American football, tears occurring in-game have been reported to most frequently happen during a

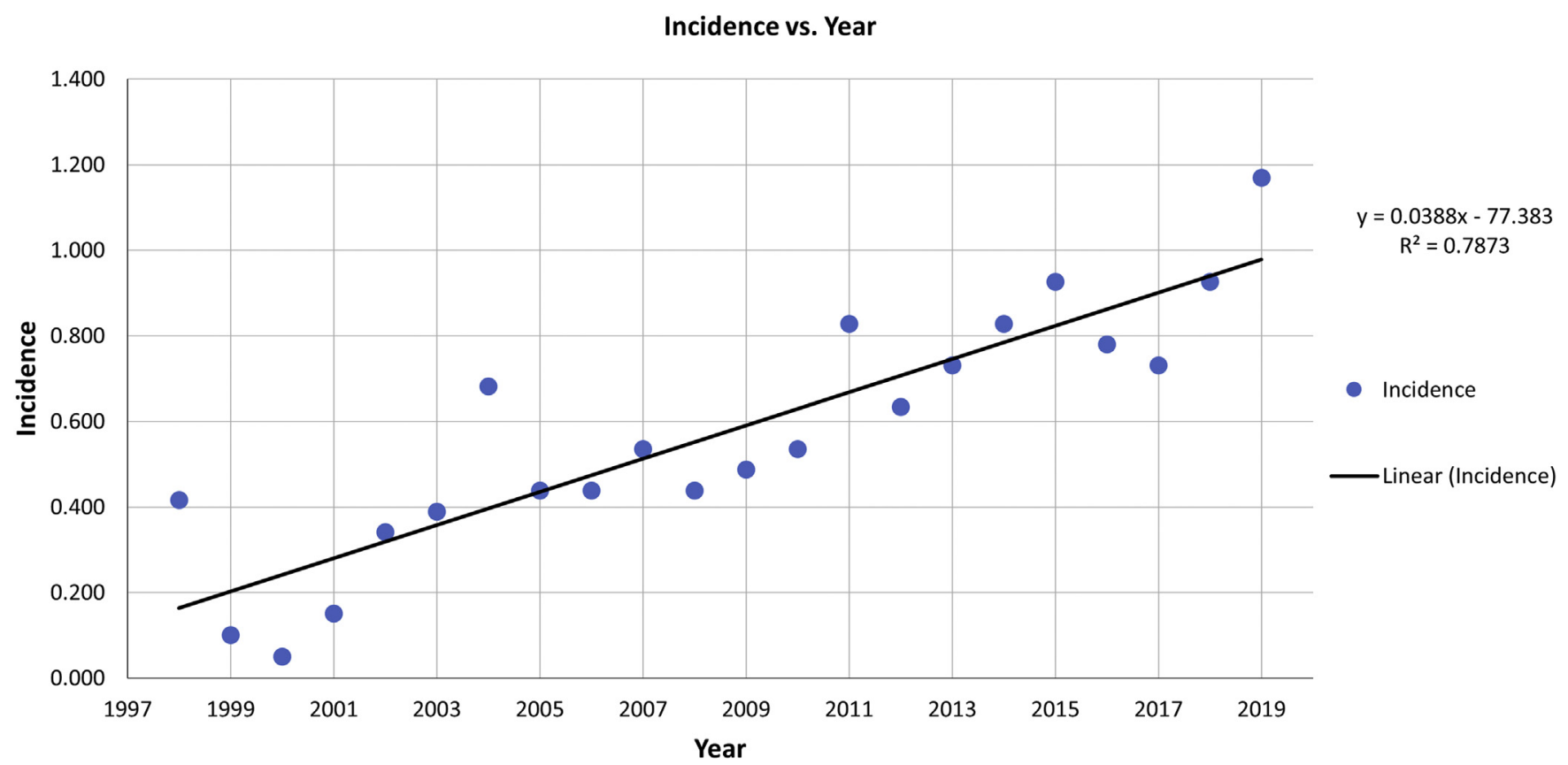

Fig. 1. Incidence of pectoralis major injury in the National Football League per year with linear regression performed. Incidence is reported as rate of injury per 10,000 athlete-exposures. 
Table 3. Management Type Based on Athlete Demographics and Position

\begin{tabular}{lc}
\hline & Mean \pm SD Where Applicable [Range] \\
\hline Conservative Management & $116(45.0 \%)$ \\
Age & $27.4 \pm 3.3$ years $[21.0-37.0]$ \\
BMI & $32.7 \pm 4.0[24.9-41.9]$ \\
Defensive athletes & $56(48.3 \%)$ \\
Defensive lineman & $21(37.5 \%)$ \\
Linebacker & $24(42.9 \%)$ \\
Defensive back/secondary & $15(26.8 \%)$ \\
Offensive athletes & $60(51.2 \%)$ \\
Offensive lineman & $30(50.0 \%)$ \\
Running back & $7(11.7 \%)$ \\
Wide receiver & $6(10.0 \%)$ \\
Tight end & $8(13.3 \%)$ \\
Quarterback & $5(8.3 \%)$ \\
Operative management & $126(48.8 \%)$ \\
Age & (26.8 0 (25) \\
BMI & $98(72.0-37.0]$ \\
Defensive athletes & $26(26.5 \%)$ \\
Defensive lineman & $46(46.9 \%)$ \\
Linebacker & $26(26.5 \%)$ \\
Defensive back/secondary & $27(21.4 \%)$ \\
Offensive athletes & $18(66.7 \%)$ \\
Offensive lineman & $5(18.5 \%)$ \\
Running back & $0(0.0 \%)$ \\
Wide receiver & $1(3.7 \%)$ \\
Tight end & $3(11.1 \%)$ \\
Quarterback & $4.0[25.3 \%)$ \\
\hline
\end{tabular}

Table 3 shows the player demographics in nonoperatively versus operatively treated players. BMI, body mass index; SD, standard deviation.

tackle. ${ }^{9,29-31}$ Miller et al. noted that the injury mechanism typically involved the arm being caught in an opponent's jersey while abducted $90^{\circ}$ and slightly externally rotated. ${ }^{30}$

Table 4. Risk Factors for Operative Pectoralis Major Tendon Injury

\begin{tabular}{lcc}
\hline \multicolumn{1}{c}{ Variables } & OR $(95 \%$ CI $)$ & $P$ Value \\
\hline Year of Injury & $1.11(1.05-1.17)$ & $<.001$ \\
Position & & \\
$\quad$ Defensive line & $3.78(1.42-10.60)$ & $\mathbf{. 0 0 9}$ \\
Defensive backs & $12.20(2.13-76.60)$ & $\mathbf{. 0 0 6}$ \\
Linebacker & $8.98(2.58-33.60)$ & $<.001$ \\
Quarterback & $2.85(.32-24.30)$ & .337 \\
Running back & $2.10(.30-13.00)$ & .432 \\
Special teams & NA & .994 \\
Tight end & $.67(.03-5.92)$ & .745 \\
Wide receiver & NA & .987 \\
Practice or game injury ${ }^{\dagger}$ & $1.65(.62-4.52)$ & .319 \\
BMI & $1.13(.98-1.32)$ & .106 \\
Age at injury & $1.24(.97-1.63)$ & .099 \\
Years of experience before injury & $.77(.59-.99)$ & $\mathbf{. 0 4 7}$ \\
\hline Table 4 demonstrated risk factors for pectoralis major injuries \\
requiring operative treatment as determined by multivariate logistic \\
regression. \\
BMI, body mass index; CI, confidence interval; OR, odds ratio. \\
Bolded $P$ values indicates statistical significance $(P<.05)$. \\
*, with reference to offensive linemen; \\
${ }^{\dagger}$, referenced to practice setting.
\end{tabular}

In the present study, defensive athletes were found to sustain a greater number of PM injuries. In American football, PM injuries have been reported to generally occur in the blocking or tackling position, when the arm is abducted, externally rotated, and extended. ${ }^{32}$ As such, this may place defensive players at greater risk for PM injuries when compared to offensive players. Specifically, the positional requirements of defensive linemen necessitate these athletes to encounter contact on each play as they attempt to move around opposing offensive linemen, forcing their arms into extension and external rotation in the process. In contrast, the high incidence of tears in linebackers and defensive backs may be attributed to the open field nature of these positions, which result in higher contact forces while the arm is in abduction during tackling or while reaching for opponents. Furthermore, the high incidence of tears during play may be explained by the high forces placed on the vulnerable arm due to the everincreasing size and speed of athletes participating at the professional level.

Surgical repair was performed in $48.8 \%$ of injuries, with increasing year at the time of injury, athletes with shorter NFL experience, as well as athletes classified as defensive linemen, defensive backs, and linebackers being predictive of operative management. Surgical repair of PM injuries is generally indicated for partial and complete tears at the tendinous insertion or musculotendinous junction or when athletes are unable to accept the resulting weakness and deformity that may occur with nonoperative management. ${ }^{14,33}$ Operative treatment of PM tendon tears has also been demonstrated to result in superior functional outcomes and strength when compared to nonoperative management. ${ }^{34}$ Generally, acute repair is favored over chronic repair, with acute repairs associated with improved functional outcome and satisfaction with cosmesis. ${ }^{35}$

\section{Limitations}

This study is not without limitations. An inherent limitation of this study is the use of public databases to capture PM injuries, as the authors identified a number of NFL injuries classified as "undisclosed" or "chest" injuries that were unable to be confirmed as PM injuries. Additionally, this study does not explore causes for the identified linear increase in PM injuries per year, as this was beyond the scope of the current investigation. Lastly, this study did not investigate return to play and performance characteristics for these injuries, but future studies from our group will.

\section{Conclusion}

A total of 258 PM injuries were identified over $22 \mathrm{NFL}$ seasons, with an overall incidence of 0.603 per 10,000 athlete-exposures, which was found to increase by 0.039 injuries per 10,000 athlete-exposures per year. 
Repair was performed in $48.8 \%$ of athletes, with more recent time of injury, shorter NFL experience, defensive linemen, defensive backs, and linebackers at significantly higher risk for operative treatment.

\section{Acknowledgment}

Investigation was performed at Midwest Orthopaedics at Rush.

\section{References}

1. Adickes MS, Stuart MJ. Youth football injuries. Sports Med 2004;34:201-207.

2. DeLee JC, Farney WC. Incidence of injury in Texas high school football. Am J Sports Med 1992;20:575-580.

3. Kretzler H Jr, Richardson A. Rupture of the pectoralis major muscle. Am J Sports Med 1989;17:453-458.

4. Liu J, Wu J, Chang C, et al. Avulsion of the pectoralis major tendon. Am J Sports Med 1992;20:366-368.

5. Zeman SC, Rosenfeld RT, Lipscomb PR. Tears of the pectoralis major muscle. Am J Sports Med 1979;7:343-347.

6. Bak K, Cameron E, Henderson I. Rupture of the pectoralis major: a meta-analysis of 112 cases. Knee Surg Sports Traumatol Arthrosc 2000;8:113-119.

7. McEntire JE, Hess WE, Coleman SS. Rupture of the pectoralis major muscle. A report of eleven injuries and review of fifty six. J Bone Jt Surg Ser A 1972;54:1040-1046.

8. Provencher $M$, Handfield $K$, Boniquit $N$, et al. Injuries to the pectoralis major muscle: diagnosis and management. Am J Sports Med 2010;38:1693-1705.

9. Schepsis A, Grafe M, Jones H, et al. Rupture of the pectoralis major muscle: Outcome after repair of acute and chronic injuries. Am J Sports Med 2000;28:9-15.

10. Wolfe S, Wickiewicz T, Cavanaugh J, et al. Ruptures of the pectoralis major muscle. An anatomic and clinical analysis. Am J Sports Med 1992;20:587-593.

11. Dick R, Ferrara MS, Agel J, et al. Descriptive epidemiology of collegiate men's football injuries: National collegiate athletic association injury surveillance system, 1988-1989 through 2003-2004. J Athl Train 2007;42:221-233.

12. Feeley BT, Kennelly S, Barnes RP, et al. Epidemiology of national football league training camp injuries from 1998 to 2007. Am J Sports Med 2008;36:1597-1603.

13. Marmor L, Bechtol CO, Hall CB. Pectoralis major muscle. Function of sternal portion and mechanism of rupture of normal muscle: Case reports. J Bone Jt Surg 1961;43: 81-87.

14. Quinlan J, Molloy M, Hurson B. Pectoralis major tendon ruptures: When to operate. Br J Sports Med 2002;36: 226-228.

15. Pro-Football-Reference.com. 2020. Available from: https://wWw.pro-football-reference.com/. Accessed March 25, 2020.

16. ESPN NFL injuries. 2020. Available from: https://www. espn.com/nfl/injuries. Accessed March 25, 2021.

17. Hsu W. Performance-based outcomes following lumbar discectomy in professional athletes in the national football league. Spine 2010;35:1247-1251.
18. Hsu W. Outcomes following nonoperative and operative treatment for cervical disc herniations in national football league athletes. Spine 2011;36:800-805.

19. Nguyen M, Hsu W. Performance-based outcomes following patellar tendon repair in professional athletes. Phys Sportsmed 2020;48:110-115.

20. Mai H, Chun D, Schneider A, et al. Performance-based outcomes after anterior cruciate ligament reconstruction in professional athletes differ between sports. Am J Sports Med 2017;45:2226-2232.

21. Minhas S, Kester B, Larkin K, et al. The effect of an orthopaedic surgical procedure in the National Basketball Association. Am J Sports Med 2016;44:1056-1061.

22. Wise P, Gallo R. Increasing incidence of pectoralis major ruptures in NFL players. Orthop J Sports Med 2019;7: 116-123.

23. NFL Football Operations. Health \& Safety Rules Changes. Available from: https://operations.nfl.com/football-ops/ nfl-ops-honoring-the-game/health-safety-rules-changes/ 2020. Accessed March 23, 2021.

24. The National Football League. 2017 Personnel (Injury) Report Policy 2017. Available from: https://operations.nfl. com/media/2683/2017-nfl-injury-report-policy.pdf.

25. Sahota S, Gibbs D, Lawton C, et al. Pectoralis major injuries in the National Football League. Sports Health 2020;12:116-123.

26. Mall N, Buchowski J, Zebala L, et al. Spine and axial skeleton injuries in the National Football League. Am J Sports Med 2012;40:1755-1761.

27. Tarity T, Garrigues G, Ciccotti M, et al. Pectoralis major ruptures in professional American football players. Phys Sportsmed 2014;42:131-135.

28. de Castro Pochini A, Ejnisman B, Andreoli C, et al. Pectoralis major muscle rupture in athletes: a prospective study. Am J Sports Med 2010;38:92-98.

29. Connell DA, Potter HG, Sherman MF, et al. Injuries of the pectoralis major muscle: Evaluation with MR imaging. Radiology 1999;210:785-791.

30. Miller MD, Johnson DL, Fu FH, et al. Rupture of the pectoralis major muscle in a collegiate football player. Use of magnetic resonance imaging in early diagnosis. Am J Sports Med 1993:21:475-477.

31. Zvijac JE, Schurhoff MR, Hechtman KS, et al. Pectoralis major tears: Correlation of magnetic resonance imaging and treatment strategies. Am J Sports Med 2006;34: 289-294.

32. Kakwani R, Matthews J, Kumar K, et al. Rupture of the pectoralis major muscle: Surgical treatment in athletes. Int Orthop 2007;31:159-163.

33. Guity M, Vaziri A, Shafiei H, et al. Surgical treatment of pectoralis major tendon rupture (outcome assessment). Asian J Sports Med 2014;5:129-135.

34. Bodendorfer B, McCormick B, Wang D, et al. Treatment of pectoralis major tendon tears: A systematic review and meta-analysis of operative and nonoperative treatment. Orthop J Sports Med 2020;8:23259671 19900813.

35. Bodendorfer B, Wang D, McCormick B, et al. Treatment of pectoralis major tendon tears: A systematic review and meta-analysis of repair timing and fixation methods. Am J Sports Med 2020;48:3376-3385. 